\title{
Computed and conventional chest radiography: A comparison of image quality and radiation dose
}

\begin{abstract}
The aim of this study was to compare the image quality and entrance skin dose (ESD) for film-screen and computed chest radiography. Analysis of the image quality and dose on chest radiography was carried out on a conventional X-ray unit using film-screen, storage phosphor plates and selenium drum direct chest radiography. For each receptor, ESD was measured in 60 patients using thermoluminescent dosemeters. Images were printed on $35 \times 43 \mathrm{~cm}$ films. Image quality was assessed subjectively by evaluation of anatomic features and estimation of the image quality, following the guidelines established by the protocols of the Commission of the European Communities. There was no statistically significant difference noted between the computed and conventional images (Wilcoxon rank sum test, P > 0.05). Imaging of the mediastinum and peripheral lung structures were better visualized with the storage phosphor and selenium drum technique than with the film-screen combination. The patients' mean ESD for chest radiography using the storage phosphor, film-screen combination and selenium drum was $0.20,0.20$ and $0.25 \mathrm{mGy}$, respectively, with no statistically significant difference with P $>0.05$ ( $\chi 2$ tests). (C) 2005 Royal Australian and New Zealand College of Radiologists
\end{abstract}

Keyword: Commission of the European Communities; Film-screen; Selenium drum; Storage phosphor 